\title{
Theory of the Circular Diffraction Antenna
}

Harold Levine and, and Charles H. Papas

Citation: Journal of Applied Physics 22, 29 (1951); doi: 10.1063/1.1699816

View online: http://dx.doi.org/10.1063/1.1699816

View Table of Contents: http://aip.scitation.org/toc/jap/22/1

Published by the American Institute of Physics

AIP| $\mid \begin{aligned} & \text { Journal of } \\ & \text { Applied Physics }\end{aligned}$

Save your money for your research.

It's now FREE to publish with us -

no page, color or publication charges apply.
Publish your research in the Joumal of Applied Physics

to claim your place in applied physics history. 
the case if the spacing is greater than or equal to a half-wavelength. If the spacing is less than a halfwavelength, only a part of one period of the space factor is available, and the case is more difficult. The fact that the the total number of roots is known will be a help, and it is conceivable that the skilled operator will be able to say much about the complete period of $\log |S|$ even though he begins by knowing only a part of it.

\section{CONCLUSIONS}

1. The linear array design problem is essentially the problem of finding a polynomial whose magnitude, when evaluated upon the unit circle, fulfills given requirements.

2. The design problem can be solved by the use of an electrical analog device which continually plots the logarithm of the magnitude of the polynomial in question in response to the operator's selection of its roots. The operator adjusts the positions of these roots in the complex plane until the output of the device is as desired. The coefficients of the polynomial, which corre- spond to the relative currents in the elements of the linear array, are then found by elementary methods.

3. The solution to the array design problem is not, in general, unique.

4. What can be done by the electrical analog can also be done by an analytical method; however, certain steps are extremely laborious. It is felt that the analog method will be more satisfactory from an engineering point of view for several reasons. For example, it is better adapted to the form in which the problem is likely to be stated, and is capable of investigating minor changes with comparative ease.

5. Closely related to the design problem is the evaluation problem which begins with an experimental determination of the magnitude of the space factor of an existing array and yields as a result the excitation coefficients of that array. This is essentially the same as the design problem as far as the mechanics of solution are concerned, and the work may be performed on the analog. Lack of uniqueness in the solution, however, will limit the utility of this technique.

\title{
Theory of the Circular Diffraction Antenna*
}

\author{
Harold Levine and Charles H. Papas \\ Cruft Laboratory, Harvard University, Cambridge, Massachusetts
}

(Received August 21, 1950)

\begin{abstract}
The circular diffraction antenna consists of a coaxial wave guide fitted with an infinite-plane conducting baffle, and open to free space. An equivalent circuit description, appropriate to principal-mode propagation in the coaxial region, is investigated theoretically. Variational expressions for the circuit parameters are derived, and used for accurate numerical evaluation.
\end{abstract}

\section{INTRODUCTION}

$T$ $\mathrm{HE}$ circular diffraction antenna (Fig. 1) is a simple system amenable to detailed theoretical analysis, and offers the possibility of close experimental comparison. For convenience in analysis, the antenna is assumed to operate with a single propagating mode (the principal mode) in the coaxial region; waves incident on the open end through the coaxial guide are excited by a remote periodic source. With a perfectly conducting structure, part of the incident energy flux on the open end is returned to the source via reflected waves of the same type, and the remainder appears in free-space waves. A useful description of the principal-mode behavior is provided by an equivalent circuit, in which the stored energy of nonpropagating modes near the open end and the energy lost by radiation are accounted for

\footnotetext{
* Part of the research reported in this document was made possible through support extended Cruft Laboratory, Harvard University, jointly by the Navy Department (ONR), the Signal Corps of the U. S. Army, and the U. S. Air Force, under ONR Contract N5ori-76, T. O. 1.
}

by lumped circuit elements. This paper is concerned with variational methods for calculating the circuit parameters. Questions which relate primarily to the radiation aspects, such as its angular distribution, are not considered.

As the principal mode of the coaxial wave guide has no angular variation in any plane normal to its propagation direction, the geometrical structure of the antenna ensures that this symmetry is characteristic of the entire electromagnetic field. A further consequence of the geometrical symmetry is that the only nonvanishing electromagnetic field components in cylindrical coordinates are $H_{\phi}, E_{\rho}, E_{z}$, the last being absent for the principal TEM mode. Since the Maxwell equations show that the steady-state electric field components are obtained from $H_{\phi}$ by differentiation, the latter serves as a basic scalar function.

The construction of field components, in accordance with the prescribed excitation and boundary condition on the conducting surfaces, is facilitated by dealing separately with coaxial and external regions. A continu- 


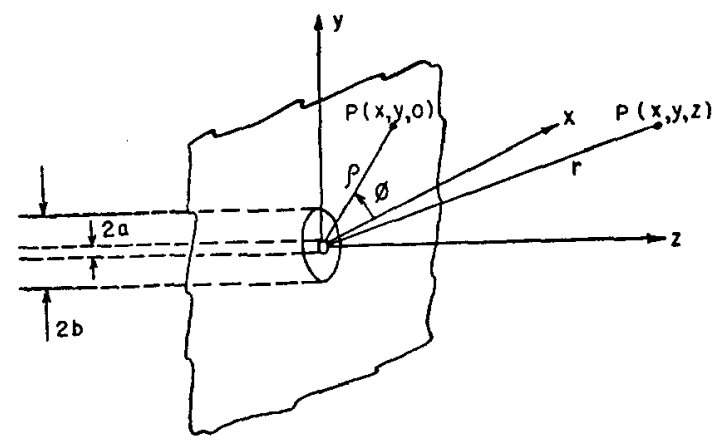

Fig. 1. Coaxial line with baffle.

ous solution for all space is contingent on equality of the respective tangential components $H_{\phi}, E_{\rho}$ in the open end of the coaxial guide (called the aperture), with continuity of the normal component $E_{z}$ then ensured. This schedule of aperture boundary conditions can be fitted into the formal development in different ways. The scalar function $H_{\phi}$ at any point in either region can be expressed in terms of integrals involving its $z$-derivative (proportional to $E_{p}$ ) in the aperture, the latter function having a null value on the conducting baffle. All continuity requirements are satisfied by equating the two functions $B_{\phi}$ at each point of the aperture, with the resulting integral equation to determine the aperture field $E_{\rho}$, and thence the electromagnetic field everywhere. Alternatively, $H_{\phi}$ in the coaxial region is given by integrals containing its aperture values, whereas the function outside is determined by these values together with the distribution on the baffle. If the $z$-derivative of the respective functions $H_{\phi}$ are set equal in the aperture, the field component $E_{\rho}$ is continuous there; moreover, the $z$-derivative of $H_{\phi}$, and hence $E_{\rho}$, is required to vanish on the baffle. Thus a pair of dual integral equations for the distribution of $B_{\phi}$ in the aperture plane is obtained.

For an engineering description, a principal-mode current and voltage in the coaxial guide are introduced. The current and voltage, in any cross section of the guide, are given by the line integrals, respectively, of the principal-mode part of $H_{\phi}$ around the inner conductor and the corresponding part of $E_{\rho}$ between inner and outer conductors; the functions thus defined satisfy uniform transmission-line equations. An equivalent circuit representation, which employs the open end of the coaxial guide as a reference plane, involves a terminal admittance for the transmission line, specified by the current-voltage ratio in the aperture. Real and imaginary parts of the admittance are measures of the radiated and stored energy, respectively.

By combining the transmission-line quantities with the integral equation formulations, a pair of variational expressions for the terminal admittance (relative to the characteristic admittance of the line) are derived. ${ }^{1}$ These

\footnotetext{
${ }^{1}$ For some of the original wave-guide applications of the variational method, see Notes on Lectures by J. Schwinger by David S. Saxon, Massachusetts Institute of Technology, February 1945.
}

have the property of being stationary with respect to variations about the aperture field $E_{\rho}$ or the field $H_{\phi}$ in the aperture plane, and can be exhibited in useful homogeneous forms. Application of the variational expressions is given, with rather satisfactory experimental comparison.

\section{FIRST INTEGRAL EQUATION FORMULATION}

In accordance with the preceding section, the electromagnetic field can be derived from a single scalar function $B_{\phi}$ which is independent of the angular coordinate $\phi$. For harmonic time dependence $\exp (-i \omega t)$ the differential equation which governs the spatial behavior of $H_{\phi}$ is

$$
\left(\frac{\partial^{2}}{\partial \rho^{2}}+\frac{1}{\rho} \frac{\partial}{\partial \rho}+\frac{\partial^{2}}{\partial z^{2}}-\frac{1}{\rho^{2}}+k^{2}\right) H_{\phi}(\rho, z)=0,
$$

where $k^{2}=\omega^{2} \epsilon \mu=2 \pi / \lambda, \omega=$ angular frequency, $\lambda=$ freespace wavelength, $\epsilon$ and $\mu$ denote respectively the dielectric constant and permeability of free space. The electric-field components are derived from $H_{\phi}$ according to the Maxwell equations

$$
\begin{gathered}
i \omega \epsilon E_{\rho}(\rho, z)=\frac{\partial}{\partial z} H_{\phi}(\rho, z), \\
-i \omega \epsilon E_{z}(\rho, z)=\left(\frac{\partial}{\partial \rho}+\frac{1}{\rho}\right) H_{\phi}(\rho, z) .
\end{gathered}
$$

In the coaxial region, $a \leq \rho \leq b, z \leq 0, H_{\phi}$ must satisfy the boundary condition

$$
\left(\frac{\partial}{\partial \rho}+\frac{1}{\rho}\right) H_{\phi}(\rho, z)=0 \text { at } \rho=a, b,
$$

which, according to (2.3), makes $E_{z}$ vanish at the surfaces of the inner and outer conductors. A general expansion for $H_{\phi}$ is

$$
\begin{aligned}
H_{\phi}^{(-)}(\rho, z)= & \alpha \frac{e^{i k z}}{\rho}+\beta \frac{e^{-i k z}}{\rho} \\
& +\sum_{n=1}^{\infty} A_{n} R_{n}(\rho) \exp \left[\left(\lambda_{n}{ }^{2}-k^{2}\right)^{\frac{1}{t}} z\right], \\
& a \leq \rho \leq b, \quad z \leq 0,
\end{aligned}
$$

where the first and second terms represent the incident and reflected TEM modes of amplitudes $\alpha$ and $\beta$ respectively and the infinite series represents the higher order modes. The eigenfunction $R_{n}(\rho)$ and the eigenvalue $\lambda_{n}$ are defined by

$$
\begin{aligned}
& \left(\frac{\partial^{2}}{\partial \rho^{2}}+\frac{1}{\rho} \frac{\partial}{\partial \rho}-\frac{1}{\rho^{2}}+\lambda_{n}^{2}\right) R_{n}(\rho)=0, \\
& \left(\frac{\partial}{\partial \rho}+\frac{1}{\rho}\right) R_{n}(\rho)=0 \quad \text { at } \rho=a, b .
\end{aligned}
$$


The eigenvalues are roots of the transcendental equation

$$
J_{0}\left(\lambda_{n} a\right) N_{0}\left(\lambda_{n} b\right)=N_{0}\left(\lambda_{n} a\right) J_{0}\left(\lambda_{n} b\right),
$$

where $J_{0}(x)$ and $N_{0}(x)$ are the Bessel and Neumann functions of order zero, and $a, b$ are the respective radii of the inner and outer conductors. Since $\lambda_{n+1}>\lambda_{n}$ and $k$ is chosen such that $k<\lambda_{1},\left(\lambda_{n}{ }^{2}-k^{2}\right)^{1}$ is real and positive. ${ }^{2}$ Consequently, all the higher order modes of (2.5) are exponentially damped with increasingly negative values of $z$. A corollary of (2.6) and (2.7) is the orthogonality of the eigenfunctions with one another and with $1 / \rho$. By an appropriate normalization

$$
\begin{gathered}
\int_{a}^{b} R_{n}(\rho) R_{m}(\rho) \rho d \rho=\delta_{n m}, \\
\int_{a}^{b} R_{n}(\rho) d \rho=0,
\end{gathered}
$$

where $\delta_{n n}=1, \delta_{n m}=0$. With the aid of (2.9) and (2.10) the coefficients $A_{n}$ of (2.5) are expressed in terms of the transverse component of the electric-field intensity in the aperture. Thus applying (2) to (5) we obtain

$$
\begin{aligned}
& i \omega \epsilon E_{\rho}(-)(\rho, z)=\frac{\partial}{\partial z} H_{\phi}^{(-)}(\rho, z)=i k \alpha \frac{e^{i k z}}{\rho}-i k \beta \frac{e^{-i k z}}{\rho} \\
& +\sum_{n=1}^{\infty} A_{n} R_{n}(\rho)\left(\lambda_{n}{ }^{2}-k^{2}\right)^{\frac{1}{2}} \\
& \times \exp \left[\left(\lambda_{n}{ }^{2}-k^{2}\right)^{\frac{1}{2}} z\right], \quad a \leq \rho \leq b, \quad z \leq 0,
\end{aligned}
$$

which, upon setting $z=0$, multiplying by $\rho R_{n}(\rho)$, and integrating from $\rho=a$ to $\rho=b$, yields

$$
A_{n}=\frac{i \omega \epsilon}{\left(\lambda_{n}^{2}-k^{2}\right)^{\frac{1}{2}}} \int_{a}^{b} \mathcal{E}(\rho) R_{n}(\rho) \rho d \rho,
$$

where $\mathcal{E}(\rho) \equiv E_{\rho}(\rho, 0)$. Substituting these $A_{n}$ 's into (2.5) we have

$$
\begin{array}{r}
H_{\phi}(-)(\rho, z)=\alpha \frac{e^{i k z}}{\rho}+\beta \frac{e^{-i k z}}{\rho}+i \omega \epsilon \int_{a}^{b} \mathcal{E}\left(\rho^{\prime}\right) \rho^{\prime} d \rho^{\prime} \\
\times \sum_{n=1}^{\infty} \frac{R_{n}(\rho) R_{n}\left(\rho^{\prime}\right)}{\left(\lambda_{n}{ }^{2}-k^{2}\right)^{\frac{1}{2}}} \exp \left[\left(\lambda_{n}{ }^{2}-k^{2}\right)^{\mathfrak{t} z}\right] \\
a \leq \rho \leq b, \quad z \leq 0,
\end{array}
$$

A direct integration of (2.11) for $z=0$ provides the relation

$$
\int_{a}^{b} \mathcal{E}(\rho) d \rho=(\alpha-\beta)\left(\frac{\mu}{\epsilon}\right)^{b} \underset{a}{\log -}
$$

Our next task is to find an expression for the azimuthal component $H_{\phi}^{(+)}(\rho, z)$ of the magnetic-field in-

\footnotetext{
${ }^{2}$ M. Kline, J. Math. Phys. 27, 37-48, No. 1 (1949); H. B
}

tensity in the half-space $z \geq 0$. For this purpose we need a Green's function $G^{(+)}\left(\rho, z, \rho^{\prime}, z^{\prime}\right)$ which satisfies the inhomogeneous equation

$$
\begin{aligned}
\left(\frac{\partial^{2}}{\partial \rho^{2}}+\frac{1}{\rho} \frac{\partial}{\partial \rho}+\frac{\partial^{2}}{\partial z^{2}}-\right. & \left.\frac{1}{\rho^{2}}+k^{2}\right) G^{(+)}\left(\rho, z, \rho^{\prime}, z^{\prime}\right) \\
& =-\delta\left(z-z^{\prime}\right) \frac{\delta\left(\rho-\rho^{\prime}\right)}{\rho}, \quad z \geq 0,
\end{aligned}
$$

and the boundary condition

$$
\frac{\partial}{\partial z} G^{(+)}\left(\rho, 0, \rho^{\prime}, z^{\prime}\right)=0 .
$$

We construct $G^{(+)}\left(\rho, \rho^{\prime}, z, z^{\prime}\right)$ by combining two solutions, one of which satisfies (2.15) for all $z$ while the other satisfies (2.15) with the sign of $z^{\prime}$ changed, such that their sum is an even function of $z$ [thereby satisfying (2.16)]. Thus

$G^{(+)}\left(\rho, z, \rho^{\prime}, z^{\prime}\right)=G\left(\rho, \rho^{\prime}, z-z^{\prime}\right)+G\left(\rho, \rho^{\prime}, z+z^{\prime}\right)$,
$z \geq 0$,

and $G\left(\rho, \rho^{\prime}, z \mp z^{\prime}\right)$ must satisfy the radiation condition and the equation

$$
\begin{aligned}
& \left(\frac{\partial^{2}}{\partial \rho^{2}}+\frac{1}{\rho} \frac{\partial}{\partial \rho}+\frac{\partial^{2}}{\partial z^{2}}-\frac{1}{\rho^{2}}+k^{2}\right) G\left(\rho, \rho^{\prime}, z \mp z^{\prime}\right) \\
& =-\delta\left(z \mp z^{\prime}\right) \frac{\delta\left(\rho-\rho^{\prime}\right)}{\rho}, \quad-\infty \leq z \leq \infty,
\end{aligned}
$$

where the upper and lower signs correspond to a source at $z=z^{\prime}, \rho=\rho^{\prime}$ and its image at $z=-z^{\prime}, \rho=\rho^{\prime}$ respectively. To determine $G\left(\rho, \rho^{\prime}, z-z^{\prime}\right)$ we take the Fourier transform ${ }^{3}$ of $(2.18)$,

$\left[\frac{\partial^{2}}{\partial \rho^{2}}+\frac{1}{\rho} \frac{\partial}{\partial \rho}-\zeta^{2}-\frac{1}{\rho^{2}}+k^{2}\right] G\left(\rho, \rho^{\prime}, \zeta\right)=\frac{-\delta\left(\rho-\rho^{\prime}\right)}{\rho}$

where $\zeta$ is a complex variable and

$$
G\left(\rho, \rho^{\prime}, \zeta\right)=\int_{-\infty}^{\infty} e^{-i \zeta z} G\left(\rho, \rho^{\prime}, z\right) d z
$$

The solution for (2.19) representing cylindrical wave propagation outwards along $\rho$ is

$$
G\left(\rho, \rho^{\prime}, \zeta\right)=\frac{\pi i}{2} H_{1}^{(1)}\left[\left(k^{2}-\zeta^{2}\right)^{\frac{1}{3}} \rho\right] J_{1}\left[\left(k^{2}-\zeta^{2}\right)^{\frac{1}{1}} \rho_{<}\right],
$$

where $\rho_{>}, \rho<$ respectively designate the larger and smaller of $\rho, \rho^{\prime}, J_{1}(x)$ is the first-order Bessel function, and $H_{1}{ }^{(\mathrm{I})}(x)=J_{1}(x)+i N_{1}(x)$ is the first-order Hankel function of the first kind. We specify the phases of the radicals in (2.20) as follows: $\arg \left(k^{2}-\zeta^{2}\right)^{3}=0, \pi / 2$ for

\footnotetext{
${ }^{3}$ H. Levine and J. Schwinger, Phys. Rev. 73, 383-406 (1948).
} 
$|\zeta|<k,|\zeta|>k$ respectively, to insure the proper sense of propagation. Applying the Fourier inversion theorem to (2.20) we obtain (Appendix A)

$$
\begin{aligned}
G\left(\rho, \rho^{\prime}, z-z^{\prime}\right) & =\frac{1}{2 \pi} \int_{-\infty}^{\infty} G\left(\rho, \rho^{\prime}, \zeta\right) e^{i \zeta\left(z-z^{\prime}\right)} d \zeta \\
& =\frac{i}{4} \int_{-\infty}^{\infty} H_{1}^{(1)}\left[\left(k^{2}-\zeta^{2}\right)^{\frac{1}{2}} \rho_{>}\right] J_{1}\left[\left(k^{2}-\zeta^{2}\right)^{\frac{1}{3}} \rho_{<}\right] e^{i \zeta\left(z-z^{\prime}\right)} d \zeta \\
& =\frac{1}{4 \pi} \int_{0}^{2 \pi} d \phi \cos \phi \frac{\exp \left\{i k\left[\rho^{2}+\rho^{\prime 2}-2 \rho \rho^{\prime} \cos \phi+\left(z-z^{\prime}\right)^{2}\right]^{\frac{1}{2}}\right\}}{\left[\rho^{2}+\rho^{\prime 2}-2 \rho \rho^{\prime} \cos \phi+\left(z-z^{\prime}\right)^{2}\right]^{\frac{1}{2}}},
\end{aligned}
$$

where the $\zeta$-integration is along the real axis of the complex $\zeta$-plane with an upward indentation at $\zeta=-k$ and a downward one at $\zeta=+k$ (Fig. 2). And by a similar procedure,

$$
G\left(\rho, \rho^{\prime}, z+z^{\prime}\right)=\frac{1}{4 \pi} \int_{0}^{2 \pi} d \phi \cos \phi \frac{\exp \left\{i k\left[\rho^{2}+\rho^{\prime 2}-2 \rho \rho^{\prime} \cos \phi+\left(z+z^{\prime}\right)^{2}\right]^{\frac{1}{3}}\right\}}{\left[\rho^{2}+\rho^{\prime 2}-2 \rho \rho^{\prime} \cos \phi+\left(z+z^{\prime}\right)^{2}\right]^{\frac{1}{2}}} .
$$

Hence, from (2.17), (2.21), and (2.22) we obtain the desired Green's function,

$$
\begin{array}{r}
G^{(+)}\left(\rho, \rho^{\prime}, z, z^{\prime}\right)=\frac{1}{4 \pi} \int_{0}^{2 \pi} d \phi \cos \phi\left[\frac{\exp \left\{i k\left[\rho^{2}+\rho^{\prime 2}-2 \rho \rho^{\prime} \cos \phi+\left(z-z^{\prime}\right)^{2}\right]^{\frac{1}{2}}\right\}}{\left[\rho^{2}+\rho^{\prime 2}-2 \rho \rho^{\prime} \cos \phi+\left(z-z^{\prime}\right)^{2}\right]^{\frac{1}{2}}}\right. \\
\left.+\frac{\exp \left\{i k\left[\rho^{2}+\rho^{\prime 2}-2 \rho \rho^{\prime} \cos \phi+\left(z+z^{\prime}\right)^{2}\right]^{\frac{1}{2}}\right\}}{\left[\rho^{2}+\rho^{\prime 2}-2 \rho \rho^{\prime} \cos \phi+\left(z+z^{\prime}\right)^{2}\right]^{\frac{1}{3}}}\right] .
\end{array}
$$

Now we apply a modified form of Green's second identity to $H_{\phi}^{(+)}(\rho, z)$ and $G^{(+)}\left(\rho, \rho^{\prime}, z, z^{\prime}\right)$ in the volume $V$ bounded by the plane $S$ of the baffle and the hemisphere $S_{\infty}$ with $r \rightarrow \infty$, (Fig. 3). Thus

$$
\begin{aligned}
\int_{V}\left\{G^{(+)}\left(\rho, \rho^{\prime}, z, z^{\prime}\right)\left[\nabla^{\prime 2}-1 / \rho^{\prime 2}+k^{2}\right] H_{\phi}^{(+)}\left(\rho^{\prime}, z^{\prime}\right)-H_{\phi}^{(+)}\left(\rho^{\prime}, z^{\prime}\right)\left[\nabla^{\prime 2}-1 / \rho^{\prime 2}+k^{2}\right] G^{(+)}\left(\rho, \rho^{\prime}, z, z^{\prime}\right)\right\} d \tau^{\prime} \\
=\int_{S+S_{\infty}}\left\{G^{(+)}\left(\rho, \rho^{\prime}, z, z^{\prime}\right) n \cdot \nabla^{\prime} H_{\phi}^{(+)}\left(\rho^{\prime}, z^{\prime}\right)-H_{\phi}^{(+)}\left(\rho^{\prime}, z^{\prime}\right) n \cdot \nabla^{\prime} G^{(+)}\left(\rho, \rho^{\prime}, z, z^{\prime}\right)\right\} d \sigma^{\prime},
\end{aligned}
$$

where $n$ is a unit vector normal to the bounding surface and directed outward from the region. The integration over $S_{\infty}$ disappears and, hence, the surface integral reduces to an integration over the plane $z=0$. By virtue of (2.1) and (2.15) the volume integration yields $H_{\phi}{ }^{(+)}(\rho, z)$; recalling (2.2), (2.16), (2.23), and the boundary condition $E_{\rho}(\rho, 0)=0, b \leq \rho \leq a,(2.24)$ becomes

$$
\begin{aligned}
H_{\phi}(+)(\rho, z) & =-\int_{S} G^{(+)}\left(\rho, \rho^{\prime}, z, 0\right) \frac{\partial}{\partial z^{\prime}} H_{\phi}^{(+)}\left(\rho^{\prime}, 0\right) \rho^{\prime} d \rho^{\prime} \\
& =\frac{-i \omega \epsilon}{2 \pi} \int_{a}^{b} \mathcal{E}\left(\rho^{\prime}\right) \rho^{\prime} d \rho^{\prime} \int_{0}^{2 \pi} d \phi \cos \phi \frac{\exp \left[i k\left(\rho^{2}+\rho^{\prime 2}-2 \rho \rho^{\prime} \cos \phi+z^{2}\right)^{\frac{1}{2}}\right]}{\left(\rho^{2}+\rho^{\prime 2}-2 \rho \rho^{\prime} \cos \phi+z^{2}\right)^{\frac{1}{2}}}, z \geq 0 .
\end{aligned}
$$

$\mathrm{U}_{\mathrm{P}}$ to this point we have obtained expressions (2.13) and (2.25) for $H_{\phi}(\rho, z)$ valid in the coaxial region $a \leq \rho \leq b, z \leq 0$ and the half-space $z \geq 0$ respectively. To proceed further we introduce the quantity of physical interest, i.e., the terminal admittance, in terms of the electric- and magnetic-field intensities.

As a measure of the coaxial-line current we take

$$
I(z)=2 \pi\left(\alpha e^{i k z}+\beta e^{-i k z}\right),
$$

which upon comparison with (2.13) is seen to be the integral of the dominant part of $H_{\phi}^{(-)}(\rho, z)$ taken along a circle of radius $\rho$ surrounding the center conductor. And as a measure of the voltage across the coaxial line we adopt the line integral of $E_{\rho}^{(-)}(\rho, z)$ from $\rho=a$ to $\rho=b$,

$$
\begin{aligned}
& V(z)=\int_{a}^{b} E_{\rho}^{(-)}(\rho, z) d \rho \\
&=\left(\frac{\mu}{\epsilon}\right)^{\frac{1}{2}}\left(\alpha e^{i k z}-\beta e^{-i k z}\right) \underset{a}{\log -},
\end{aligned}
$$




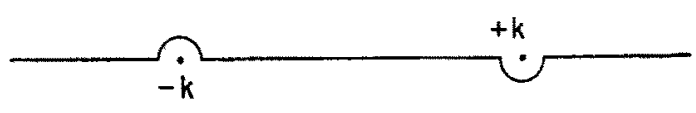

FIG. 2. Path of integration in complex $\zeta$-plane.

which follows from (2.11) and the orthogonality relation (2.10). In particular

$$
V(0)=\int_{a}^{b} \mathcal{E}(\rho) d \rho=\left(\frac{\mu}{\epsilon}\right)^{\frac{1}{2}}(\alpha-\beta) \underset{a}{b}
$$

and

$$
I(0)=2 \pi(\alpha+\beta)
$$

The current and voltage as defined by (2.26) and (2.27) satisfy the uniform transmission-line equations,

$$
\begin{gathered}
(\partial / \partial z) I(z)=i k Y_{0} V(z), \\
(\partial / \partial z) V(z)=i k Z_{0} I(z),
\end{gathered}
$$

where $Y_{0}\left(=1 / Z_{0}\right)$ is the characteristic admittance and is given by

$$
Y_{0}=\frac{2 \pi}{(\mu / \epsilon)^{\frac{1}{2}} \log (b / a)}
$$

Fig. 3. Region of integration. $S=$ plane of baffle. $S_{\infty}=$ hemisphere with $r=\infty . V=$ volume enclosed by $S_{\infty}+S$.

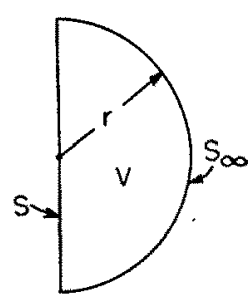

The admittance $Y(z)$ at any cross section is given by the current-voltage ratio $Y(z)=I(z) / V(z)$. Taking the open end of the coaxial line as a reference plane we obtain an equivalent-circuit representation consisting of a lumped admittance $Y(0)$ terminating the transmission line. From (2.28), (2.29), and (2.32) we see that the terminal admittance is given by

$$
Y(0)=\frac{I(0)}{V(0)}=\frac{\alpha+\beta}{\alpha-\beta}
$$

A knowledge of $Y(0)$ offers a complete description of the dominant-mode behavior.

Returning to the main thread of the discussion we see that $H_{\phi}^{(-)}(\rho, z)$ as given by (2.13) can be rewritten with the aid of (2.26) as

$$
H_{\phi}^{(-)}(\rho, z)=\frac{I(z)}{2 \pi \rho}+i \omega \epsilon \int_{a}^{b} \mathscr{E}\left(\rho^{\prime}\right) \rho^{\prime} d \rho^{\prime} \sum_{n=1}^{\infty} \frac{R_{n}(\rho) R_{n}\left(\rho^{\prime}\right)}{\left(\lambda_{n}{ }^{2}-k^{2}\right)^{\frac{1}{2}}} \exp \left[\left(\lambda_{n}{ }^{2}-k^{2}\right)^{\frac{1}{z}} z\right], \quad z \leq 0 .
$$

Across the aperture $H_{\phi}(\rho, z)$ must be continuous, i.e., $H_{\phi}^{(\rightarrow)}(\rho, 0)=H_{\phi}^{(+)}(\rho, 0)$. Consequently setting $z=0$ in $(2.25)$ and (2.34) we obtain the desired integral equation

$$
\begin{array}{rl}
\frac{I(0)}{2 \pi \rho}+i \omega \epsilon \int_{a}^{b} & \mathcal{E}\left(\rho^{\prime}\right) \rho^{\prime} d \rho^{\prime} \sum_{n=1}^{\infty} \frac{R_{n}(\rho) R_{n}\left(\rho^{\prime}\right)}{\left(\lambda_{n}{ }^{2}-k^{2}\right)^{\frac{1}{2}}} \\
& =\frac{-i \omega \epsilon}{2 \pi} \int_{a}^{b} \mathcal{E}\left(\rho^{\prime}\right) \rho^{\prime} d \rho^{\prime} \int_{0}^{2 \pi} d \phi \cos \phi \frac{\exp \left[i k\left(\rho^{2}+\rho^{\prime 2}-2 \rho \rho^{\prime} \cos \phi\right)^{\frac{1}{2}}\right]}{\left(\rho^{2}+\rho^{\prime 2}-2 \rho \rho^{\prime} \cos \phi\right)^{\frac{1}{2}}}, \quad a \leq \rho \leq b .
\end{array}
$$

\section{SECOND INTEGRAL EQUATION FORMULATION}

Using an alternative existence theorem, which allows the representation of the electromagnetic field within a region in terms of the tangential magnetic field on the bounding surface, we now deduce a pair of dual equations for the distribution of the magnetic-field intensity $\mathfrak{F C}(\rho)$ in the aperture plane.

We recall from (2.5) that

$$
H_{\phi}(-)(\rho, z)=\frac{e^{i k z}}{\rho}+\beta \frac{e^{-i k z}}{\rho}+\sum_{n=1}^{\infty} A_{n} R_{n}(\rho) \exp \left[\left(\lambda_{n}^{2}-k^{2}\right)^{\frac{1}{2} z}\right], \quad a \leq \rho \leq b, \quad z \leq 0 .
$$

Multiplying by $\rho R_{n}(\rho)$, then integrating from $\rho=a$ to $\rho=b$, we obtain by (2.9), (2.10) for $z=0$

$$
A_{m}=\int_{a}^{b} \mathfrak{H}(\rho) R_{m}(\rho) \rho d \rho,
$$

where $\mathfrak{H C}(\rho) \equiv H_{\phi}^{(-)}(\rho, 0)$. Similarly by a direct integration of $(2.5)$

$$
\int_{a}^{b} \pi(\rho) d \rho=(\alpha+\beta) \underset{a}{\log -=}=\frac{I(0)}{2 \pi} \log -\frac{b}{a}
$$


where (2.29) was used to establish the latter equality. Substituting (3.1) into (2.5) we find

$$
H_{\phi}^{(-)}(\rho, z)=\frac{e^{i k z}}{\rho}+\beta \frac{e^{-i k z}}{\rho}+\int_{a}^{b} \mathcal{H}\left(\rho^{\prime}\right) \rho^{\prime} d \rho^{\prime} \sum_{n=1}^{\infty} R_{n}(\rho) R_{n}\left(\rho^{\prime}\right) \exp \left[\left(\lambda_{n}^{2}-k^{2}\right) z\right], \quad a \leq \rho \leq b, \quad z \leq 0,
$$

which, when differentiated according to $(2.2)$ yields

$$
\begin{aligned}
& E_{\rho}(-)(\rho, z)=\left(\frac{\mu}{-}\right)^{\frac{1}{2}}\left(\frac{e^{i k z}}{\alpha-\beta-\frac{e^{-i k z}}{\rho}}\right) \\
& +\frac{1}{i \omega \epsilon} \int_{a}^{b} \mathfrak{K}\left(\rho^{\prime}\right) \rho^{\prime} d \rho^{\prime} \sum_{n=1}^{\infty} R_{n}(\rho) R_{n}\left(\rho^{\prime}\right)\left(\lambda_{n}{ }^{2}-k^{2}\right)^{\frac{1}{2}} \exp \left[\left(\lambda_{n}^{2}-k^{2}\right)^{\frac{1}{2}} z\right], \quad a \leq \rho \leq b, \quad z \leq 0 .
\end{aligned}
$$

To derive an expression for the transverse component $E_{\rho}{ }^{(+)}(\rho, z)$ of the electric-field intensity for the half-plane $z \geq 0$, we construct a Green's function $\Gamma\left(\rho, \rho^{\prime}, z, z^{\prime}\right)$ which satisfies the same differential equation (2.15) as before, but with a different boundary condition,

Thus

$$
\Gamma\left(\rho, \rho^{\prime}, 0, z^{\prime}\right)=0
$$

$\Gamma\left(\rho, \rho^{\prime}, z, z^{\prime}\right)=G\left(\rho, \rho^{\prime}, z-z^{\prime}\right)-G\left(\rho, \rho^{\prime}, z+z^{\prime}\right)$

$$
\begin{aligned}
=\frac{1}{4 \pi} \int_{0}^{2 \pi} d \phi \cos \phi\left[\frac{\exp \left\{i k\left[\rho^{2}+\rho^{\prime 2}-2 \rho \rho^{\prime} \cos \phi+\left(z-z^{\prime}\right)^{2}\right]^{1}\right\}}{\left[\rho^{2}+\rho^{\prime 2}-2 \rho \rho^{\prime} \cos \phi+\left(z+z^{\prime}\right)^{2}\right]^{\frac{1}{2}}}\right. \\
\left.\quad-\frac{\exp \left\{i k\left[\rho^{2}+\rho^{\prime 2}-2 \rho \rho^{\prime} \cos \phi+\left(z+z^{\prime}\right)^{2}\right]^{1}\right\}}{\left[\rho^{2}+\rho^{\prime 2}-2 \rho \rho^{\prime} \cos \phi+\left(z+z^{\prime}\right)^{2}\right]^{\frac{1}{2}}}\right] .
\end{aligned}
$$

From Green's identity (2.24), with $G^{(+)}\left(p, \rho^{\prime}, z, z^{\prime}\right)$ replaced by $\Gamma\left(\rho, \rho^{\prime}, z, z^{\prime}\right)$, it follows that

$$
H_{\phi}^{(+)}(\rho, z)=\int_{0}^{\infty} H_{\phi}^{(+)}\left(\rho^{\prime}, 0\right) \frac{\partial}{\partial z^{\prime}} \Gamma\left(\rho, \rho^{\prime}, z, 0\right) \rho^{\prime} d \rho^{\prime}, \quad z \geq 0,
$$

from which we get, with the aid of (2.2),

where $\mathfrak{T C}(\rho) \equiv H_{\phi}^{(+)}(\rho, 0)$.

$$
E_{\rho}{ }^{(+)}(\rho, z)=\frac{1}{i \omega \epsilon} \int_{0}^{\infty} \mathcal{K}\left(\rho^{\prime}\right) \frac{\partial}{\partial z} \frac{\partial}{\partial z^{\prime}} \Gamma\left(\rho, \rho^{\prime}, z, 0\right) \rho^{\prime} d \rho^{\prime}, \quad z \geq 0,
$$

In the aperture $E_{p}^{(-)}(\rho, 0)=E_{\rho}{ }^{(+)}(\rho, 0)$. Hence by $(3.3)$ and $(3.7)$

$$
\begin{aligned}
\left(\begin{array}{c}
\mu \\
\epsilon
\end{array}\right)^{\frac{1}{2}}\left(\begin{array}{cc}
\alpha & \beta \\
- & -
\end{array}\right)+\frac{1}{i \omega \epsilon} \int_{\alpha}^{b} \mathfrak{H}\left(\rho^{\prime}\right) \rho^{\prime} d \rho^{\prime} \sum_{n=1}^{\infty} R_{n}(\rho) R_{n}\left(\rho^{\prime}\right)\left(\lambda_{n}^{2}-k^{2}\right)^{\frac{1}{2}} & =\frac{1}{i \omega \epsilon} \int_{0}^{\infty} \mathfrak{K}\left(\rho^{\prime}\right) \frac{\partial}{\partial z} \frac{\partial}{\partial z^{\prime}} \Gamma\left(\rho, \rho^{\prime}, 0,0\right) \rho^{\prime} d \rho^{\prime}, \quad a \leq \rho \leq b,
\end{aligned}
$$

and on the remainder of the plane $E_{\rho}{ }^{(+)}(\rho, 0)$ must vanish:

$$
0=\int_{0}^{\infty} \pi \mathcal{H}\left(\rho^{\prime}\right) \frac{\partial}{\partial z} \frac{\partial}{\partial z^{\prime}} \Gamma\left(\rho, \rho^{\prime}, 0,0\right) \rho^{\prime} d \rho^{\prime}, \quad a \geq \rho \geq b .
$$

Multiplying (3.8) and (3.9) by $\rho \mathfrak{F C}(\rho)$ and integrating,

$$
\begin{aligned}
\left(\frac{\mu}{\epsilon}\right)^{t}(\alpha-\beta) \int_{a}^{b} \mathfrak{H}(\rho) d \rho+\frac{1}{i \omega \epsilon} \sum_{n=1}^{\infty}\left(\lambda_{n}{ }^{2}-k^{2}\right)^{3}\left[\int_{a}^{b} \mathcal{H}(\rho) R_{n}(\rho) \rho d \rho\right]^{2} & \\
& =\frac{1}{i \omega \epsilon} \int_{0}^{\infty} \rho d \rho \mathcal{H}(\rho) \int_{0}^{\infty} \rho^{\prime} d \rho^{\prime} \mathcal{H}\left(\rho^{\prime}\right) \frac{\partial}{\partial z} \frac{\partial}{\partial z^{\prime}} \Gamma\left(\rho, \rho^{\prime}, 0,0\right) .
\end{aligned}
$$


We rewrite (3.10) in a form involving the terminal voltage $V(0)$ given by (2.28):

$$
\begin{aligned}
\frac{V(0)}{\log (b / a)} \int_{a}^{b} \mathfrak{H}(\rho) d \rho+\frac{1}{i \omega \epsilon} \sum_{n=1}^{\infty}\left(\lambda_{n}{ }^{2}-k^{2}\right)^{3}\left[\int_{a}^{b} \mathfrak{H}(\rho) R_{n}(\rho) \rho d \rho\right]^{2} & \\
& =\frac{1}{i \omega \epsilon} \int_{0}^{\infty} \rho d \rho \mathcal{H C}(\rho) \int_{0}^{\infty} \rho^{\prime} d \rho^{\prime} \mathcal{H C}\left(\rho^{\prime}\right) \frac{\partial}{\partial z} \frac{\partial}{\partial z^{\prime}} \Gamma\left(\rho, \rho^{\prime}, 0,0\right) .
\end{aligned}
$$

\section{VARIATIONAL PRINCIPLES}

Utilizing the first integral equation, we construct a variational principle for $Y(0)$ by first multiplying (2.35) by $\rho \mathcal{E}(\rho)$, then integrating from $\rho=a$ to $\rho=b$, and finally dividing the resultant equation by $\left[\int_{a}^{b} \mathcal{E}(\rho) d \rho\right]^{2}$. Thus, recalling that $V(0)=\int_{a}^{b} \mathcal{E}(\rho) d_{\rho}(2.28)$ and $Y(0)=I(0) / V(0)(2.33)$, we obtain the desired representation,

$$
\begin{aligned}
& \frac{Y(0)}{Y_{0}}=\frac{-i k \underset{a}{b}-}{\left[\int_{a}^{b} \mathcal{E}(\rho) d \rho\right]^{2}}\left\{\sum_{n=1}^{\infty} \frac{\left[\int_{a}^{b} \mathcal{E}(\rho) R_{n}(\rho) \rho d \rho\right]^{2}}{\left(\lambda_{n}{ }^{2}-k^{2}\right)^{\frac{1}{2}}}\right. \\
& \left.+\frac{1}{2 \pi} \int_{a}^{b} \rho d \rho \int_{a}^{b} \rho^{\prime} d \rho^{\prime} \int_{0}^{2 \pi} d \phi \cos \phi \mathcal{E}(\rho) \mathcal{E}\left(\rho^{\prime}\right) \frac{\exp \left[i k\left(\rho^{2}+\rho^{\prime 2}-2 \rho \rho^{\prime \prime}, \cos \phi\right)^{\frac{1}{2}}\right]}{\left(\rho^{2}+\rho^{\prime 2}-2 \rho \rho^{\prime} \cos \phi\right)^{\frac{1}{2}}}\right\}
\end{aligned}
$$

for $Y(0) / Y_{0}$ in terms of the aperture electric field $\mathcal{E}(\rho)$, which is homogeneous in $\mathcal{E}(\rho)$ and stationary with respect to the first variation of $\mathcal{E}(\rho)$ about its correct value determined by $(2.35)$.

Another variational principle is deduced by dividing (3.11) by $\left[\int_{a}^{b} \mathfrak{F C}(\rho) d \rho\right]^{2}$. Thus, since $2 \pi \mathcal{S}_{a}^{b} \mathcal{H C}(\rho) d \rho=I(0)$ $\times \log b / a, Y(0)=I(0) / V(0)$,

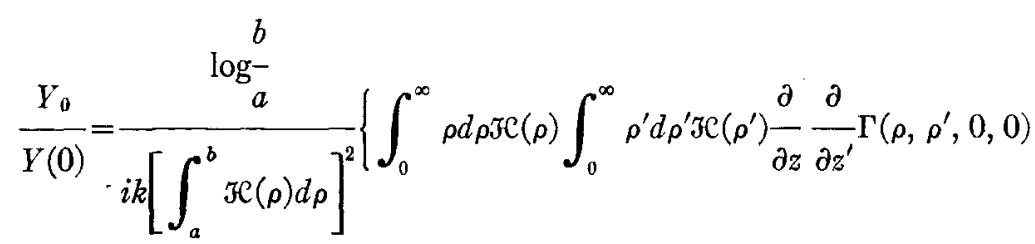

$$
\begin{aligned}
& -\sum_{n=1}^{\infty}\left(\lambda_{n}{ }^{2}-k^{2}\right)^{2}\left\{\left[\int_{a}^{b} \mathcal{H}(\rho) R_{n}(\rho) \rho d \rho\right]^{2}\right\},
\end{aligned}
$$

which is homogeneous in $\mathfrak{H C}(\rho)$ and stationary with respect to first variations about its correct value determined by (3.11).

In both variational principles the stationary quantity, $Y(0)=G(0)-i B(0)$ where $G(0)$ and $B(0)$ are the terminating conductance and susceptance respectively, is complex. If we substitute the true $\mathcal{E}(\rho)$ in $(4.1)$ and the true $\mathfrak{H}(\rho)$ in (4.2), obtained from (2.35) and (3.11), respectively, both variational principles will yield the same true value for $Y(0)$. However, the two values of $Y(0)$ resulting from approximate $\mathcal{E}(\rho)$ and $\mathscr{H}(\rho)$ will differ, but both will be close to the true $Y(0)$ if the trial functions are chosen appropriately. The errors in $Y(0)$ are of the order of magnitude of the squares of the errors in $\mathcal{E}(\rho)$ and $\mathscr{H}(\rho)$.

If the stationary quantity had not been complex, one variational principle would yield a value above the true one while the other would yield a value below it. Since $Y(0)$ is complex no such limits can be found.

\section{APPLICATION OF THE VARIATIONAL PRINCIPLES}

We evaluate $Y(0)$ using first (4.1) involving $\mathcal{E}(\rho)$ and then (4.2) involving $\mathscr{H}(\rho)$. Assuming

$$
\mathcal{E}(\rho)=\sum_{n=0}^{\infty} a_{n} R_{n}(\rho)
$$

where $R_{0}(\rho)=1 / \rho$, we calculate the integrals of (4.1) in terms of $a_{n}$. Recalling the orthogonality properties (2.9), 
(2.10) of $R_{n}(\rho)$, we directly get

and

$$
\left[\int_{a}^{b} \mathcal{E}(\rho) d \rho\right]^{2}=\left[\sum_{n=0}^{\infty} a_{n} \int_{a}^{b} R_{n}(\rho) d \rho\right]^{2}=a_{0}^{2}(\underset{a}{\log -})^{2}
$$

$$
\left[\int_{a}^{b} \mathcal{E}(\rho) R_{n}(\rho) \rho d \rho\right]^{2}=\left[\sum_{m=0}^{\infty} a_{m} \int_{a}^{b} R_{m}(\rho) R_{n}(\rho) \rho d \rho\right]^{2}=\left[\sum_{m=0}^{\infty} a_{m} \delta_{m n}\right]^{2}=a_{n}{ }^{2}
$$

The remaining integral of (4.1) is cast into a more convenient form by the relation (Appendix A)

$$
\frac{1}{2 \pi} \int_{0}^{2 \pi} d \phi \cos \phi \frac{\exp \left[i k\left(\rho^{2}+\rho^{\prime 2}-2 \rho \rho^{\prime} \cos \phi\right)^{\frac{1}{2}}\right]}{\left(\rho^{2}+\rho^{\prime 2}-2 \rho \rho^{\prime} \cos \phi\right)^{\frac{1}{2}}}=\int_{0}^{\infty} \frac{\zeta d \zeta}{\left(\zeta^{2}-k^{2}\right)^{\frac{1}{2}}} J_{1}(\zeta \rho) J_{1}\left(\zeta \rho^{\prime}\right)
$$

where the $\zeta$-integration is along the real axis of the complex $\zeta$-plane with a downward indentation at $\zeta=+k$ and $\arg \left(\zeta^{2}-k^{2}\right)^{\frac{1}{2}}=0,-\pi / 2$ for $\zeta \geq k, \zeta \leq k$ respectively. Thus

where

$$
\frac{1}{2 \pi} \int_{a}^{b} \rho d \rho \int_{a}^{b} \rho^{\prime} d \rho^{\prime} \int_{0}^{2 \pi} d \phi \cos \phi \mathcal{E}(\rho) \mathcal{E}\left(\rho^{\prime}\right) \frac{\exp \left[i k\left(\rho^{2}+\rho^{\prime 2}-2 \rho \rho^{\prime} \cos \phi\right)^{\frac{1}{2}}\right]}{\left(\rho^{2}+\rho^{\prime 2}-2 \rho \rho^{\prime} \cos \phi\right)^{\frac{1}{2}}}=\sum_{n=0}^{\infty} \sum_{m=0}^{\infty} a_{n} a_{m} c_{n m},
$$

$$
c_{n m}=\int_{a}^{\infty} \frac{\zeta d \zeta}{\left(\zeta^{2}-k^{2}\right)^{\frac{1}{2}}} \int_{a}^{b} \rho d \rho \int_{0}^{b} \rho^{\prime} d \rho^{\prime} J_{1}(\zeta \rho) J_{1}\left(\zeta \rho^{\prime}\right) R_{n}(\rho) R_{m}\left(\rho^{\prime}\right)=c_{m n} .
$$

Carrying out the $\rho$ and $\rho^{\prime}$ integrations of (5.6) (Appendix B),

where

$$
c_{n m}=\int_{0}^{\infty} \frac{\zeta d \zeta}{\left(\zeta^{2}-k^{2}\right)^{\frac{1}{2}}} f_{n}(\zeta) f_{m}(\zeta)
$$

and

and

$$
\begin{aligned}
f_{n}(\zeta) & =\int_{a}^{b} \rho d \rho R_{n}(\rho) J_{1}(\zeta \rho) \\
& =\frac{2}{\pi} \frac{N_{n}}{\lambda_{n}} \frac{1}{J_{0}\left(\lambda_{n} b\right)} \frac{\zeta}{\lambda_{n}{ }^{2}-\zeta^{2}}\left[J_{0}(\zeta b) J_{0}\left(\lambda_{n} a\right)-J_{0}(\zeta a) J_{0}\left(\lambda_{n} b\right)\right],
\end{aligned}
$$

$$
f_{0}(\zeta)=\int_{a}^{b} \rho d \rho R_{0}(\rho) J_{1}(\zeta \rho)=\frac{J_{0}(\zeta a)-J_{0}(\zeta b)}{\zeta}
$$

$$
\frac{1}{N_{n}}=\frac{\sqrt{2}}{\pi \lambda_{n}}\left[\frac{J_{0}^{2}\left(\lambda_{n} a\right)}{J_{0}^{2}\left(\lambda_{n} b\right)}-1\right]^{\frac{1}{2}}
$$

Inserting (5.2), (5.3), and (5.5) into (4.1) we get the following expression for $Y(0)$ in terms of the coefficients $a_{m}$ (only the relative amplitudes $a_{m} / a_{0}$ are important for the calculation):

$$
\frac{Y(0)}{Y_{0}}=\frac{-i k}{\log (b / a)}\left\{\sum_{n=1}^{\infty} \frac{\bar{a}_{n}^{2}}{\left(\lambda_{n}{ }^{2}-k^{2}\right)^{\frac{1}{2}}}+\sum_{n=1}^{\infty} \sum_{m=1}^{\infty} \bar{a}_{n} \bar{a}_{m} c_{n m}+2 \sum_{m=1}^{\infty} \bar{a}_{m} c_{0 m}+c_{00}\right\},
$$

where $\bar{a}_{m}=a_{m} / a_{0}$.

Owing to the stationary character of $Y(0)$, on differentiating (5.11) with respect to $\bar{a}_{n}$, we obtain the following infinite set of simultaneous equations,

$$
\frac{\bar{a}_{n}}{\left(\lambda_{n}{ }^{2}-k^{2}\right)^{\frac{1}{2}}}+c_{0 n}+\sum_{m=1}^{\infty} \bar{a}_{m} c_{n m}=0, \quad n=1,2,3, \cdots, \infty,
$$

which, when multiplied by $\bar{a}_{n}$ and summed on $n$, yield

$$
\sum_{n=1}^{\infty} \frac{\bar{a}_{n}^{2}}{\left(\lambda_{n}^{2}-k^{2}\right)^{\frac{1}{2}}}+\sum_{n=1}^{\infty} \bar{a}_{n} c_{0 n}+\sum_{n=1}^{\infty} \sum_{m=1}^{\infty} \bar{a}_{n} \bar{a}_{m} c_{n m}=0 .
$$


As a consequence of $(5.13),(5.11)$ becomes

$$
\frac{Y(0)}{Y_{0}}=\frac{-i k}{\log (b / a)}\left\{c_{00}+\sum_{m=1}^{\infty} \bar{a}_{m} c_{0 m}\right\}
$$

Now we proceed with the evaluation of $Y(0)$ using (4.2). In preparation for this, we deduce an appropriate representation for $\mathfrak{K C}(\rho)$. From $(2.25)$ and $(5.4)$,

$$
H_{\phi}^{(+)}(\rho, z)=-i \omega \epsilon \int_{0}^{\infty} \frac{\zeta d \zeta}{\left(\zeta^{2}-k^{2}\right)^{\frac{1}{2}}} J_{\mathfrak{l}}(\zeta \rho) \int_{a}^{\delta} \rho^{\prime} d \rho^{\prime} J_{1}\left(\zeta \rho^{\prime}\right) \mathcal{E}\left(\rho^{\prime}\right) .
$$

Inserting the expansion (5.1) into (5.15) we find for $\mathscr{K}(\rho)$ (apart from scale factors)

$$
\mathfrak{H C}(\rho)=\sum_{m=0}^{\infty} a_{m} \int_{0}^{\infty} \frac{\zeta d \zeta}{\left(\zeta^{2}-k^{2}\right)^{\frac{1}{3}}} J_{1}(\zeta \rho) f_{m}(\zeta)
$$

since $\mathfrak{F}(\rho) \equiv H_{\phi}^{(+)}(\rho, 0)$. From (5.9) and (5.16)

from which it follows that

$$
\begin{aligned}
\int_{a}^{b} 3 C(\rho) d \rho & =\sum_{m=0}^{\infty} a_{m} \int_{0}^{\infty} \frac{\zeta d \zeta}{\left(\zeta^{2}-k^{2}\right)^{\frac{1}{3}}} f_{m}(\zeta) \int_{a}^{b} J_{1}(\zeta \rho) d \rho \\
& =\sum_{m=0}^{\infty} a_{m} \int_{0}^{\infty} \frac{\zeta d \zeta}{\left(\zeta^{2}-k^{2}\right)^{\frac{\zeta}{3}}} f_{m}(\zeta) f_{0}(\zeta)=\sum_{m=0}^{\infty} a_{m} c_{0 m}
\end{aligned}
$$

Since [Appendix A(13)]

$$
\left[\int_{a}^{b} \mathfrak{x}(\rho) d \rho\right]^{2}=\left(\sum_{m=0}^{\infty} a_{m} c_{0 m}\right)^{2}
$$

we write

$$
\frac{\partial}{\partial z} \frac{\partial}{\partial z^{\prime}} \Gamma\left(\rho, \rho^{\prime}, 0,0\right)=-\int_{0}^{\infty} \zeta\left(\zeta^{2}-k^{2}\right)^{\xi} J_{1}(\zeta \rho) J_{1}\left(\zeta \rho^{\prime}\right) d \zeta
$$

$$
\int_{0}^{\infty} \rho d \rho \mathcal{H}(\rho) \int_{0}^{\infty} \rho^{\prime} d \rho^{\prime} \mathcal{H}\left(\rho^{\prime}\right) \frac{\partial}{\partial z} \frac{\partial}{\partial z^{\prime}} \Gamma\left(\rho, \rho^{\prime}, 0,0\right)=-\int_{0}^{\infty} \zeta d \zeta\left(\zeta^{2}-k^{2}\right)^{\frac{1}{2}}\left[\int_{0}^{\infty} \rho d \rho \mathcal{H}(\rho) J_{1}(\zeta \rho)\right]^{2},
$$

and proceed to evaluate $\int_{0}^{\infty} \rho d \rho \mathcal{H}(\rho) J_{1}(\zeta \rho)$. Utilizing (5.16), we get

$$
\int_{0}^{\infty} \rho d \rho \mathcal{H}(\rho) J_{1}(\zeta \rho)=\int_{0}^{\infty} \rho d \rho J_{1}(\zeta \rho) \sum_{m=0}^{\infty} a_{m} \int_{0}^{\infty} \frac{\xi d \xi}{\left(\xi^{2}-k^{2}\right)^{\frac{1}{3}}} J_{1}(\xi \rho) f_{m}(\xi)
$$

where $\xi$ is a complex variable. Recognizing that

(5.21) becomes

$$
\int_{0}^{\infty} \rho d \rho J_{1}(\zeta \rho) J_{1}(\xi \rho)=\frac{\delta(\zeta-\xi)}{\zeta}
$$

$$
\int_{0}^{\infty} \rho d \rho \operatorname{re}(\rho) J_{1}(\zeta \rho)=\sum_{m=0}^{\infty} a_{m} \frac{f_{m}(\zeta)}{\left(\zeta^{2}-k^{2}\right)^{\frac{1}{2}}}
$$

It follows from (5.7), (5.20), (5.23) that

$$
\begin{aligned}
\int_{0}^{\infty} \rho d \rho \mathfrak{H}(\rho) \int_{0}^{\infty} \rho^{\prime} d \rho^{\prime} \mathfrak{H}\left(\rho^{\prime}\right) \frac{\partial}{\partial z} \frac{\partial}{\partial z^{\prime}} \Gamma\left(\rho, \rho^{\prime}, 0,0\right) & \\
& =-\int^{\infty} \zeta d \zeta\left(\zeta^{2}-k^{2}\right) \sum_{n=0}^{\infty} \sum_{m=0}^{\infty} \frac{a_{n} a_{m}}{\zeta^{2}-k^{2}} f_{n}(\zeta) f_{m}(\zeta)=\sum_{n=0}^{\infty} \sum_{m=0}^{\infty} a_{n} a_{m} c_{n m}
\end{aligned}
$$


Next we evaluate $\int_{a}^{b} \mathcal{F C}(\rho) R_{n}(\rho) \rho d \rho$. Again, from (5.16) we get

$$
\int_{a}^{\zeta} \Im(\rho) R_{n}(\rho) \rho d \rho=\sum_{m=0}^{\infty} a_{m} \int_{0}^{\infty} \frac{\zeta d \zeta}{\left(\zeta^{2}-k^{2}\right)^{\frac{1}{2}}} f_{m}(\zeta) \int_{a}^{b} \rho d \rho R_{n}(\rho) J_{1}(\zeta \rho)
$$

which by (5.7) and (5.8) becomes

$$
\int_{a}^{b} \mathfrak{H}(\rho) R_{n}(\rho) \rho d \rho=\sum_{m=0}^{\infty} a_{m} c_{n m}
$$

With the aid of (5.18), (5.24), and (5.26) we get for (4.2)

$$
\frac{Y_{0}}{Y(0)}=\frac{\log -\sum_{a}^{\infty} \sum_{n=0}^{\infty} a_{n} a_{m} c_{n m}+\sum_{m=1}^{\infty}\left(\lambda_{n}^{2}-k^{2}\right)^{\frac{1}{2}}\left(\sum_{m=0}^{\infty} a_{m} c_{n m}\right)^{2}}{-i k} .
$$

This expression is stationary and upon differentiating it with respect to $a_{p}$ we obtain

$$
c_{0 p} \sum_{m=0}^{\infty} a_{m} c_{0 m}=\left(\frac{Y(0)}{Y_{0}} \frac{\log (b / a)}{-i k}\right)\left(\sum_{m=0}^{\infty} a_{m} c_{p m}+\sum_{n=1}^{\infty} c_{p n}\left(\lambda_{n}{ }^{2}-k^{2}\right)^{\frac{1}{1}} \sum_{m=0}^{\infty} a_{m} c_{m n}\right), \quad p=0,1,2,3, \cdots, \infty .
$$

Then

where

$$
c_{0 p} \sum_{m=0}^{\infty} a_{m} c_{0 m}=\left(\frac{Y(0)}{Y_{0}} \frac{\log (b / a)}{-i k}\right) \sum_{m=0}^{\infty} a_{m} d_{p m}
$$

We now define $b_{n}$ by the relation

$$
d_{p m}=c_{p m}+\sum_{n=1}^{\infty} c_{p n} c_{m n}\left(\lambda_{n}^{2}-k^{2}\right)^{\frac{1}{1}}=d_{m p}
$$

$$
a_{n}=\frac{Y_{0}}{Y(0)} \frac{-i k}{\log (b / a)} b_{n} \sum_{m=0}^{\infty} a_{m} c_{0 m}
$$

from which, after multiplication by $c_{0 n}$ and summation on $n$, it follows that

$$
\sum_{n=0}^{\infty} a_{n} c_{0 n}=\frac{Y_{0}}{Y(0)} \frac{-i k}{\log (b / a)} \sum_{n=0}^{\infty} b_{n} c_{0 n} \sum_{n=0}^{\infty} a_{m} c_{0 m} .
$$

The sum $\sum_{n=0}^{\infty} a_{n} c_{0 n}$ is common to both sides of (5.32); canceling it out we obtain

$$
\frac{Y(0)}{Y_{0}}=\frac{-i k}{\log (b / a)} \sum_{m=0}^{\infty} b_{n} c_{0 n}
$$

Inserting (5.31) into (5.28) and using ${ }^{-}(5.33)$, we get the following equations for the $b_{n}$ :

$$
c_{0 p}=\sum_{m=0}^{\infty} b_{m} d_{p m}, \quad p=0,1,2,3, \cdots, \infty
$$

The first and second variational principles have yielded (5.14) and (5.33) where the determination of the associated coefficients $\bar{a}_{n}, b_{n}$ require the solution of two infinite sets of simultaneous equations (5.12) and (5.34). A rigorous solution is not attempted; instead we assume that $\bar{a}_{n}=b_{n}=0$ when $n \geq N$. The corresponding approximations to $Y(0)$ are given by

where

$$
\frac{Y(0)}{Y_{0}}=\frac{-i k}{\log (b / a)}\left\{c_{00}+\sum_{n=1}^{N} \bar{a}_{n} c_{0 n}\right\}
$$

$$
\frac{\bar{a}_{n}}{\left(\lambda_{n}^{2}-k^{2}\right)^{\frac{3}{2}}}+c_{\hat{0} n}+\sum_{m=1}^{\infty} \bar{a}_{m} c_{n m}=0, \quad n=1,2,3, \cdots, N \text {, }
$$


for the first variational principle, and

where

$$
\frac{Y(0)}{Y_{0}}=\frac{-i k}{\log (b / a)} \sum_{n=0}^{N} b_{n} c_{0 n}
$$

$$
\sum_{n=0}^{N} b_{n} d_{p n}=c_{0 p}, \quad p=0,1,2, \cdots, N
$$

for the second.

The computational procedure indicated in (5.35)-(5.38) affords approximations to any desired degree. The simplest approximation (and yet a sufficiently accurate one, as we shall see upon comparison with experimental results) is obtained by neglecting all but the leading terms. Hence from (5.35) we obtain

and from (5.37)

$$
\frac{Y(0)}{Y_{0}}=\frac{-i k}{\log (b / a)} \int_{0}^{\infty} \frac{d \zeta}{\zeta\left(\zeta^{2}-k^{2}\right)^{\frac{1}{2}}}\left[J_{0}(\zeta a)-J_{0}(\zeta b)\right]^{2},
$$

$$
\frac{Y(0)}{Y_{0}}=\frac{-i k}{\log (b / a)} \cdot \frac{\left(\int_{0}^{\infty} \frac{d \zeta}{\zeta\left(\zeta^{2}-k^{2}\right)^{\frac{1}{2}}}\left[J_{0}(\zeta a)-J_{0}(\zeta b)\right]^{2}\right)^{2}}{\int_{0}^{\infty} \frac{d \zeta}{\zeta\left(\zeta^{2}-k^{2}\right)^{\frac{1}{3}}}\left[J_{0}(\zeta a)-J_{0}(\zeta b)\right]^{2}+\sum_{n=1}^{\infty}\left(\lambda_{n}{ }^{2}-k^{2}\right)^{\frac{1}{2}}\left\{\int_{0}^{\infty} \frac{d \zeta}{\left(\zeta^{2}-k^{2}\right)^{\frac{1}{2}}}\left[J_{0}(\zeta a)-J_{0}(\zeta b)\right] f_{n}(\zeta)\right\}^{2}},
$$

where $f_{n}(\zeta)$ is given by (5.8).

If the approximation were improved by employing larger values of $N$ in (5.35) and (5.37), the difference between the two resulting values for $Y(0)$ would progressively decrease and in the limit $N \rightarrow \infty$ both variational principles would yield the same true value. Comparing (5.39) with (5.40) it is seen that they differ by virtue of the term,

$$
\sum_{n=1}^{\infty}\left(\lambda_{n}{ }^{2}-k^{2}\right)^{\frac{1}{2}}\left\{\int_{0}^{\infty} \frac{d \zeta}{\left(\zeta^{2}-k^{2}\right)^{\frac{1}{2}}}\left[J_{0}(\zeta a)-J_{0}(\zeta b)\right] f_{n}(\zeta)\right\}^{2},
$$

the magnitude of which offers a measure of accuracy for (5.39). That this term is small is placed in evidence by the fact that (5.41) converges at least as fast as $1 / n^{3}$ (this directly follows from (5.41) and the asymptotic relation $\lambda_{n} \sim n \pi /(b-a)$ as $\left.n \rightarrow \infty\right)$ and by the close comparison between $Y(0)$ computed from (5.39) and experiment.

\section{EVALUATION OF THE CIRCUIT PARAMETERS †}

The terminating conductance $G(0)$ and susceptance $B(0)$ are obtained from the real and imaginary parts of $Y(0)$ since $Y(0)=G(0)-i B(0)$. Thus, from (5.39)

$$
\begin{aligned}
\frac{Y(0)}{Y_{0}}=\frac{G(0)}{Y_{0}}-i \frac{B(0)}{Y_{0}}=\frac{-i k}{\log (b / a)} \int_{0}^{\infty} \frac{d \zeta}{\zeta\left(\zeta^{2}-k^{2}\right)^{\frac{1}{2}}} \\
\times\left[J_{0}(\zeta a)-J_{0}(\zeta b)\right]^{2} .
\end{aligned}
$$

The path of integration along the positive real axis of the complex $\zeta$-plane with a downward indentation at $\zeta=k$ is considered as the sum of three separate integrations: one extending from $\zeta=0$ to $\zeta=k-\eta$, another along the semicircular indentation of radius $\eta$, and finally one from $\zeta=k+\eta$ to $\zeta=\infty$. As $\eta \rightarrow 0$ the integral along the semicircle vanishes. Therefore, (6.1) is the sum of two integrals, one extending from $\zeta=0$ to $\zeta=k$, the other from $\zeta=k$ to $\zeta=\infty$; using the transformations

$\dagger$ The analytical results of this section were obtained by one of us (H.L.) at the Radiation Laboratory, Massachusetts Institute of Technology, 1945 and are contained in the Wave Guide Hand$b o o k$, Vol. 10 of the Laboratory Series (McGraw-Hill Book Company, Inc., New York). $\zeta=k \sin \theta$ and $\zeta=k \xi$ in the former and latter respectively, we obtain

$$
\begin{aligned}
\frac{G(0)}{Y_{0}}=\frac{1}{\log (b / a)} \int_{0}^{\pi / 2} & \frac{d \theta}{\sin \theta} \\
& \times\left[J_{0}(k a \sin \theta)-J_{0}(k b \sin \theta)\right]^{2}, \\
\frac{B(0)}{Y_{0}}= & \frac{1}{\log (b / a)} \int_{1}^{\infty} \frac{d}{\xi\left(\xi^{2}-1\right)^{\frac{1}{2}}}\left[J_{0}(k a \xi)-J_{0}(k b \xi)\right]^{2} .
\end{aligned}
$$

With the aid of the relations (Appendix C)

$$
\begin{aligned}
& \int_{z}^{\infty} \frac{J_{0}(k a \xi) J_{0}(k b \xi)}{\xi\left(\xi^{2}-z^{2}\right)^{\frac{1}{2}}} d \xi \\
& =\frac{\pi}{2 z}-\frac{1}{\pi z} \int_{0}^{\pi} \operatorname{Si}\left[k z\left(a^{2}+b^{2}-2 a b \cos \psi\right)^{\frac{1}{2}}\right] d \psi \\
& \int_{z}^{\infty} \frac{J_{0}^{2}(k a \xi)}{\xi\left(\xi^{2}-z^{2}\right)^{\frac{1}{2}}} d \xi=\frac{\pi}{2 z}-\frac{1}{\pi z} \int_{0}^{\pi} \operatorname{Si}(2 k a z \sin \psi / 2) d \psi
\end{aligned}
$$


FIG. 4. Terminal conductance and susceptance (relative to characteristic admittance) as function of $k a, \tau=b / a . k=2 \pi \div$ free [space wavelength.
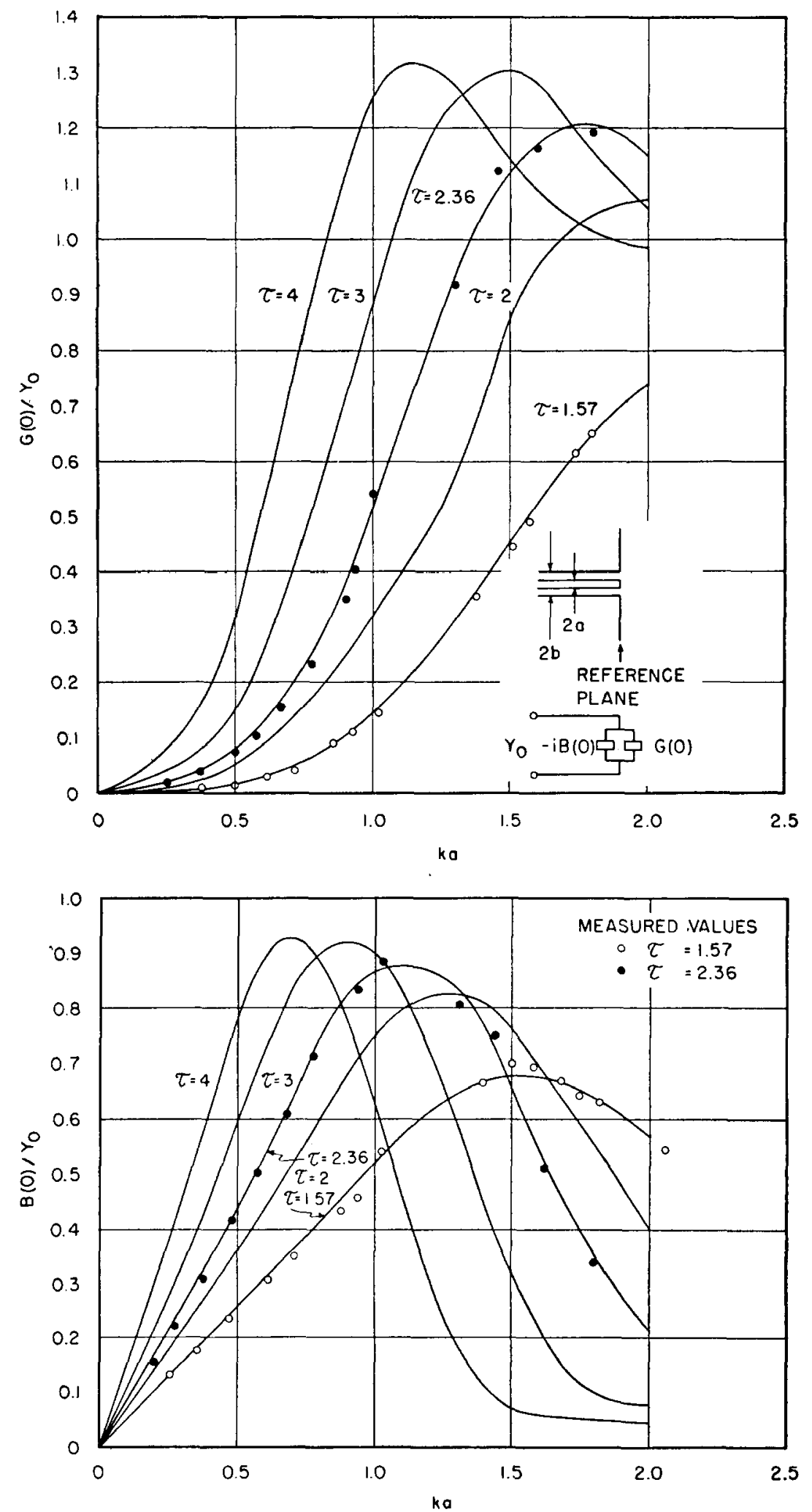
where

$$
S i(x)=\int_{0}^{x} \frac{\sin t}{t} d t
$$

is the sine integral, (6.3) takes the more convenient form:

$$
\begin{aligned}
\frac{B(0)}{Y_{0}}= & \frac{1}{\pi \log (b / a)} \\
& \times 2 \int_{0}^{\pi} \operatorname{Si}\left\{k a\left[1+(b / a)^{2}-2(b / a) \cos \psi\right]^{\frac{1}{2}}\right\} d \psi \\
& -\int_{0}^{\pi} \operatorname{Si}(2 k a \sin \psi / 2) d \psi \\
& \quad-\int_{0}^{\pi} \operatorname{Si}(2 k b \sin \psi / 2) d \psi .
\end{aligned}
$$

From (6.2) and (6.6) $G(0) / Y_{0}$ and $B(0) / Y_{0}$ have been computed for various values of $k a$ with $\tau=b / a=1.57$, $2.0,2.36,3.0$, and 4.0 and compared with experimental values $^{4}$ obtained for $\tau=1.57$ and 2.36 . In Fig. 4 it is seen that the agreement between the calculated and measured values is satisfactory.

In the low frequency limit, i.e., $k a, k b \ll 1$, we obtain from (6.2) by retaining only the first two terms in the power series development of the Bessel functions

$$
\frac{G(0)}{Y_{0}} \simeq \frac{2}{3 \log \tau}\left(\frac{1}{2}\right)^{4}\left(\tau^{2}-1\right)^{2},
$$

where $\tau=b / a$. Moreover, since $\operatorname{Si}(x) \simeq x$ when $x \ll 1$, at low frequencies (6.6) reduces to

$$
\begin{aligned}
& \frac{B(0)}{Y_{0}}=\frac{1}{\pi \log (b / a)} \\
& \times\left\{2 k a \int_{0}^{\pi}\left[1+\left(\frac{b}{a}\right)^{2}-2\left(\frac{b}{a}\right) \cos \psi\right]^{\frac{1}{2}} d \psi-4(k a+k b)\right\} .
\end{aligned}
$$

In terms of the complete elliptic integral of the second kind, $E(\alpha)$, defined by

$$
E(\alpha)=\int_{0}^{\pi / 2}\left(1-\alpha^{2} \sin ^{2} \phi\right)^{\frac{1}{2}} d \phi,
$$

(6.8) takes the form

$$
\begin{aligned}
\frac{B(0)}{Y_{0}}=\frac{4 k(a+b)}{\pi \log (b / a)}\left\{E\left[\frac{2(a b)^{\frac{1}{2}}}{a+b}\right]-1\right\} & \\
& =\frac{4 k a(1+\tau)}{\pi \log \tau}\left\{E\left[\frac{2(\tau)^{\frac{1}{2}}}{\tau+1}\right]-1\right\},
\end{aligned}
$$

4 E. O. Hartig, thesis, Harvard University, 1950. which is valid for $k a, k b \ll 1$. Furthermore, when $\alpha^{2} \equiv\left[2(\tau)^{\frac{1}{2}} / \tau+1\right]^{2} \simeq 1$ or $\tau \simeq 1$,

$$
E(\alpha) \simeq 1+\frac{1}{2}\left[\log \frac{4}{\left(1-\alpha^{2}\right)^{\frac{1}{2}}}-\frac{1}{2}\right]\left(1-\alpha^{2}\right) .
$$

Consequently, for $k a, k b \ll 1, \tau \simeq 1$ we obtain from (6.10) and (6.11)

$$
\frac{B(0)}{Y_{0}}=\frac{2 k a}{\pi \log \tau}\left[\log 4-\log \frac{\tau-1}{\tau+1}-\frac{1}{2}\right] .
$$

Finally, we may note that in the limit $k a, k b \rightarrow \infty$

$$
\begin{array}{r}
\frac{G(0)}{Y_{0}}-i \frac{B(0)}{Y_{0}}=\frac{-i k}{\log (b / a)} \int_{0}^{\infty} \frac{d \zeta}{\zeta\left(\zeta^{2}-k^{2}\right)^{\frac{1}{2}}}\left[J_{0}(\zeta a)-J_{0}(\zeta b)\right]^{2} \\
\simeq \frac{1}{\log (b / a)} \int_{0}^{\infty} \frac{d x}{x}\left[J_{0}(x)-J_{0}(x \tau)\right]^{2}=1, \quad(6.13)
\end{array}
$$

where the transformations $x=\zeta a,\left(\zeta^{2}-k^{2}\right)^{\frac{1}{2}} \rightarrow-i k$ are effected in going from the first integral to the second. Hence, in the high frequency limit $G(0) \simeq Y_{0}$ and $B(0) \simeq 0$ and the antenna is matched to free space for there is no reflection of the dominant mode wave back into the coaxial guide.

\section{ACKNOWLEDGMENT}

The authors wish to thank Miss Lesley C. Gerould and Miss Suzanne Knight for performing the numerical calculations.

\section{APPENDIX A}

In spherical coordinates the solution of

$$
\left(\nabla^{2}+k^{2}\right) G\left(r, r^{\prime}\right)=-\delta\left(r-r^{\prime}\right),
$$

where $r, r^{\prime}$ are vectors extending from the origin to the point of observation and the point source respectively, is

$$
G\left(r, r^{\prime}\right)=\frac{e^{i k\left|r-r^{\prime}\right|}}{4 \pi\left|r-r^{\prime}\right|}
$$

Physically, (2) represents spherical waves emanating from the point $r=r^{\prime}$. In cylindrical coordinates (1) becomes

$$
\left(\nabla^{2}+k^{2}\right) G\left(\rho, \rho^{\prime}, \phi-\phi^{\prime}, z-z^{\prime}\right)=\frac{-\delta\left(\rho-\rho^{\prime}\right)}{\rho} \delta\left(\phi-\phi^{\prime}\right) \delta\left(z-z^{\prime}\right) .
$$

It has been shown in Sec. IV, Eq. (13) of reference 3 that (3) has the solution

$G\left(\rho, \rho^{\prime}, \phi-\phi^{\prime}, z-z^{\prime}\right)=\frac{i}{8 \pi} \int_{-\infty}^{\infty} \sum_{m=-\infty}^{m=\infty} \exp \left[i m\left(\phi-\phi^{\prime}\right)\right] H_{m}{ }^{(1)}$

$$
\times\left[\left(k^{2}-\zeta^{2}\right)^{\frac{1}{2}} \rho>\right] J_{m}\left[\left(k^{2}-\zeta^{2}\right)^{\frac{1}{2}} \rho_{<}\right] \exp \left[i \zeta\left(z-z^{\prime}\right)\right] d \zeta,
$$

where the integration path is shown in Fig. 2. Since (2) and (4) are alternative representations, we have

$$
\begin{aligned}
\frac{e^{i k\left|r-r^{\prime}\right|}}{4 \pi\left|r-r^{\prime}\right|}= & \frac{i}{8 \pi} \int_{-\infty}^{\infty} \sum_{m=-\infty}^{\infty} \exp \left[i m\left(\phi-\phi^{\prime}\right)\right] H_{m}(1) \\
& \times\left[\left(k^{2}-\zeta^{2}\right)^{\frac{1}{\rho}} \rho>\right] J_{m}\left[\left(k^{2}-\zeta^{2}\right)^{\frac{1}{2}} \rho_{<}\right] \exp \left[i \zeta\left(z-z^{\prime}\right)\right] d \zeta,
\end{aligned}
$$

where $\quad\left|r-r^{\prime}\right|=\left(\rho^{2}+\rho^{\prime 2}-2 \rho \rho^{\prime} \cos \left(\phi-\phi^{\prime}\right)+\left(z-z^{\prime}\right)^{2}\right)^{\prime}$. Setting $\phi^{\prime}=0$ and integrating from $\phi=0$ to $\phi=2 \pi$ after multiplication by 
$\cos \phi$, we have

$\frac{1}{4 \pi} \int_{0}^{2 \pi} \frac{e^{i k\left|-r^{\prime}\right|}}{\left|r-r^{\prime}\right|} \cos \phi d \phi=\frac{i}{4} \int_{-\infty}^{\infty} H_{1}^{(1)}$

$\times\left[\left(k^{2}-\zeta^{2}\right)^{\frac{1}{2}} \rho>J_{1}\left[\left(k^{2}-\zeta^{2}\right)^{\frac{1}{2}} \rho<\right] \exp \left[i \zeta\left(z-z^{\prime}\right)\right] d \zeta\right.$,

since

$$
\int_{0}^{2 \pi} \cos m \phi \cos n \phi d \phi=\pi \delta_{n m}
$$

By definition

$$
\begin{aligned}
& \Gamma\left(\rho, \rho^{\prime}, z, z^{\prime}\right)=\frac{1}{4 \pi} \int_{0}^{2 \pi} d \phi \cos \phi \\
& \quad \times\left\{\frac{\exp \left\{i k\left[R^{2}+\left(z-z^{\prime}\right)^{2}\right]^{\frac{1}{2}}\right\}}{\left[R^{2}+\left(z-z^{\prime}\right)^{2}\right]^{3}}-\frac{\exp \left\{i k\left[R^{2}+\left(z+z^{\prime}\right)^{2}\right]^{j}\right\}}{\left[R^{2}+\left(z+z^{\prime}\right)^{2}\right]^{3}}\right\},
\end{aligned}
$$

where $R^{2}=\rho^{2}+\rho^{\prime 2}-2 \rho \rho^{\prime} \cos \phi$. Owing to Sommerfeld's representation $^{5}$

$$
\begin{aligned}
\frac{\exp \left\{i k\left[R^{2}+\left(z \pm z^{\prime}\right)^{2}\right]^{\sharp}\right\}}{\left[R^{2}+\left(z \pm z^{\prime}\right)^{2}\right]^{3}}= & \int_{0}^{\infty} \frac{\zeta d \zeta}{\left(\zeta^{2}-k^{2}\right)^{\natural}} \\
& \times \exp \left[-\left(\zeta^{2}-k^{2}\right)^{\ddagger}\left|z \pm z^{\prime}\right|\right] J_{0}(R \zeta),
\end{aligned}
$$

where the integration is along the real axis of the complex $\zeta$-plane with a downward indentation at $\zeta=+k$ and $\arg \left(\zeta^{2}-k^{2}\right)^{i}=0$, $-\pi / 2$ for $\zeta>k, \zeta<k$ respectively, we can rewrite (7) in the following form:

$$
\begin{aligned}
& \Gamma\left(\rho, \rho^{\prime}, z, z^{\prime}\right)=\frac{1}{4 \pi} \int_{0}^{2 \pi} d \phi \cos \phi \int_{0}^{\infty} \frac{\zeta d \zeta}{\left(\zeta^{2}-k^{2}\right)^{\frac{2}{2}}} J_{0}(R \zeta) \\
& \quad \times\left\{\exp \left[-\left(\zeta^{2}-k^{2}\right)^{\prime}\left|z-z^{\prime}\right|\right]-\exp \left[-\left(\zeta^{2}-k^{2}\right)^{\prime}\left|z+z^{\prime}\right|\right]\right\} .
\end{aligned}
$$

Then,

$\frac{\partial}{\partial z} \frac{\partial}{\partial z^{\prime}} \Gamma\left(\rho, \rho^{\prime}, z, z^{\prime}\right)=-\frac{1}{4 \pi} \int_{0}^{2 \pi} d \phi \cos \phi \int_{0}^{\infty} \zeta\left(\zeta^{2}-k^{2}\right)^{3} J_{0}(R \zeta)$

$$
\times\left\{\exp \left[-\left(\zeta^{2}-k^{2}\right)^{\frac{1}{2}}\left|z-z^{\prime}\right|\right]+\exp \left[-\left(\zeta^{2}-k^{2}\right)^{\frac{1}{2}}\left|z+z^{\prime}\right|\right]\right\} .
$$

At $z=z^{\prime}=0$,

$\frac{\partial}{\partial z} \frac{\partial}{\partial z^{\prime}} \boldsymbol{\Gamma}\left(\rho, \rho^{\prime}, 0,0\right)=-\frac{1}{2 \pi} \int_{0}^{2 \pi} d \phi \cos \phi \int_{0}^{\infty} \zeta\left(\zeta^{2}-k^{2}\right)^{1} J_{0}(R \zeta)$.

Interchanging the order of integration and using the addition theorem,

$$
J_{0}(R \zeta)=\sum_{n=0}^{\infty}\left(2-\delta_{0 n}\right) J_{n}(\zeta \rho) J_{n}\left(\zeta \rho^{\prime}\right) \cos n \phi
$$

we obtain

$\int_{0}^{2 \pi} d \phi \cos \phi J_{0}(R \zeta)$

$$
\begin{aligned}
& =\sum_{n=0}^{\infty}\left(2-\delta_{0 n}\right) J_{n}(\zeta \rho) J_{n}\left(\zeta \rho^{\prime}\right) \int_{0}^{2 \pi} \cos \phi \cos n \phi d \phi \\
& =2 \pi J_{1}(\zeta \rho) J_{1}\left(\zeta \rho^{\prime}\right) .
\end{aligned}
$$

Hence, by virtue of (12), (11) takes the form

$$
\frac{\partial}{\partial z} \frac{\partial}{\partial z^{\prime}} \Gamma\left(\rho, \rho^{\prime}, 0,0\right)=-\int_{0}^{\infty} \zeta\left(\zeta^{2}-k^{2}\right)^{1} J_{1}(\zeta \rho) J_{1}\left(\zeta \rho^{\prime}\right) d \zeta .
$$

Setting $z=z^{\prime}=0$ in (8), we get

$$
\frac{e^{i k R}}{R}=\int_{0}^{\infty} \frac{\zeta d \zeta}{\left(\zeta^{2}-k^{2}\right)^{\frac{3}{3}}} J_{0}(R \zeta)
$$

Multiplying through by $\cos \phi$ and integrating from $\phi=0$ to $\phi=2 \pi$, (14) becomes

$$
\begin{aligned}
\int_{0}^{2 \pi} \frac{e^{i k R}}{R} \cos \phi d \phi=\int_{0}^{\infty} \frac{\zeta d \zeta}{\left(\zeta^{2}-k^{2}\right)^{\frac{3}{3}}} \int_{0}^{2 \pi} J_{0}(R \zeta) \cos \phi d \phi \\
=2 \pi \int_{0}^{\infty} \frac{\zeta d \zeta}{\left(\zeta^{2}-k^{2}\right)^{\frac{1}{2}}} J_{1}(\zeta \rho) J_{1}\left(\zeta \rho^{\prime}\right) .
\end{aligned}
$$

\footnotetext{
${ }^{5}$ Watson, Theory of Bessel Functions (Cambridge University
} Press, London, 1945).

\section{APPENDIX B}

We consider

$$
f_{n}(\zeta)=\int_{a}^{b} \rho d \rho J_{1}(\zeta \rho) R_{n}(\rho), \quad n \geq 1 .
$$

To carry out the evaluation of $f_{n}(\zeta)$, we first must determine the normalization factor $N_{n}$ for the eigenfunction $R_{n}(\rho)$ :

$$
R_{n}(\rho)=N_{n}\left[J_{1}\left(\lambda_{n} \rho\right) N_{0}\left(\lambda_{n} a\right)-J_{0}\left(\lambda_{n} a\right) N_{1}\left(\lambda_{n} \rho\right)\right],
$$

where $\lambda_{n}$ are roots of

$$
N_{0}\left(\lambda_{n} a\right) J_{0}\left(\lambda_{n} b\right)=J_{0}\left(\lambda_{n_{t}} a\right) N_{0}\left(\lambda_{n} b\right) .
$$

$N_{n}$ are chosen such that

$$
\int_{a}^{b} R_{n}(\rho) R_{m}(\rho) \rho d \rho=\delta_{n m} .
$$

Let

$$
Z_{p}=J_{p}\left(\lambda_{n} \rho\right) N_{0}\left(\lambda_{n} a\right)-J_{0}\left(\lambda_{n} a\right) N_{p}\left(\lambda_{n} \rho\right) .
$$

Then (4) becomes

$$
\begin{aligned}
N_{n} \int_{a}^{b}\left[Z_{1}\left(\lambda_{n} \rho\right)\right]^{2} \rho d \rho & =1 \\
& =N_{n}^{2}\left\{\frac{\rho^{2}}{2}\left(Z_{1}^{2}\left(\lambda_{n} \rho\right)-Z_{0}\left(\lambda_{n} \rho\right) Z_{2}\left(\lambda_{n} \rho\right)\right)\right\}_{a}^{b} .
\end{aligned}
$$

With the aid of the identity $J_{1}(x) N_{0}(x)-J_{0}(x) N_{1}(x)=2 / \pi x$ and (3), it follows from (6) that

$$
\frac{1}{N_{n}}=\frac{\sqrt{2}}{\pi \lambda_{n}}\left[\frac{J_{0}^{2}\left(\lambda_{n} a\right)}{J_{0}^{2}\left(\lambda_{n} b\right)}-1\right]^{3} \text {. }
$$

Substituting (2) into (1) and noting that

$\int_{a}^{b} \rho d \rho J_{1}(\zeta \rho) J_{1}\left(\lambda_{n} \rho\right)$

$$
=\left.\frac{\zeta \rho J_{1}\left(\lambda_{n} \rho\right) J_{0}(\zeta \rho)-\lambda_{n} \rho J_{0}\left(\lambda_{n} \rho\right) J_{1}(\zeta \rho)}{\lambda_{n}^{2}-\zeta^{2}}\right|_{\rho=a} ^{\rho=b},
$$

and

$$
\begin{aligned}
\int_{a}^{b} \rho d \rho J_{1}(\zeta \rho) N_{1}\left(\lambda_{n} \rho\right) & \\
& =\left.\frac{\zeta \rho N_{1}\left(\lambda_{n} \rho\right) J_{0}(\zeta \rho)-\lambda_{n} \rho N_{0}\left(\lambda_{n} \rho\right) J_{1}(\zeta \rho)}{\lambda_{n}^{2}-\zeta^{2}}\right|_{\rho=a} ^{\rho=b},
\end{aligned}
$$

we finally get:

$$
\begin{aligned}
f_{n}(\zeta) & =\int_{a}^{b} \rho d \rho J_{1}(\zeta \rho) R_{n}(\rho) \\
& =\frac{2}{\pi} \frac{N_{n}}{\lambda_{n}} \frac{1}{J_{0}\left(\lambda_{n} b\right)} \frac{\zeta}{\lambda_{n}{ }^{2}-\zeta^{2}}\left[J_{0}(\zeta b) J_{0}\left(\lambda_{n} a\right)-J_{0}(\zeta a) J_{0}\left(\lambda_{n} b\right)\right],
\end{aligned}
$$

when $n=0$,

$$
f_{0}(\zeta)=\int_{a}^{b} \rho d \rho J_{1}(\zeta \rho) R_{0}(\rho)=\int_{a}^{b} d \rho J_{1}(\zeta \rho)=\frac{J_{0}(\zeta a)-J_{0}(\zeta b)}{\zeta} .
$$

\section{APPENDIX C}

Sonine's formula (p. 417, Eq. 5, reference 5)

$$
\int_{0}^{\infty} \frac{J_{\nu}\left[a\left(t^{2}+z^{2}\right)^{3}\right]}{\left(l^{2}+z^{2}\right)^{\nu / 2}} t^{2 \mu+1} d t=\frac{2^{\mu} \Gamma(\mu+1)}{a^{\mu+1} z^{\nu-\mu-1}} J_{\nu-\mu-1}(a z),
$$

is valid provided $a \geq 0, \operatorname{Re}\left(\frac{1}{2} \nu-\frac{1}{4}\right), \operatorname{Re}(\mu)>-1$, where $\operatorname{Re}$ denotes the real part.

If we let $\xi=\left(t^{2}+z^{2}\right)^{3}$ it follows from (1) that

$$
\int_{z}^{\infty} \frac{J_{\nu}(a \xi)}{\xi^{\nu-1}}\left(\xi^{2}-z^{2}\right)^{\mu} d \xi=\frac{2^{\mu} \Gamma(\mu+1)}{a^{\mu+1} z^{\nu-\mu-1}} J_{\nu-\mu-1}(a z) .
$$

We now consider the integral

$$
\begin{aligned}
F(a, b, z) \equiv \int_{z}^{\infty} \frac{J_{0}(a \xi) J_{0}(b \xi)}{\xi\left(\xi^{2}-z^{2}\right)^{1}} d \xi \\
=\frac{1}{\pi} \int_{0}^{\pi} d \theta \int_{z}^{\infty} \frac{J_{0}\left[\xi\left(a^{2}+b^{2}-2 a b \cos \theta\right)^{\dagger}\right]}{\xi\left(\xi^{2}-z^{2}\right)^{\frac{1}{2}}} d \xi,
\end{aligned}
$$

where use is made of Neumann's addition theorem and the 
orthogonality of the trigonometric functions in passing from the first integral definition to the second.

Let

$$
G(a, b, z)=\int_{z}^{\infty} \frac{J_{0}\left[\xi\left(a^{2}+b^{2}-2 a b \cos \theta\right)^{2}\right]}{\xi\left(\xi^{2}-z^{2}\right)^{k}} d \xi .
$$

Then

$$
\begin{aligned}
\frac{\partial}{\partial a} G(a, b, z)=-\frac{a-b \cos \theta}{\left(a^{2}+b^{2}-2 a b \cos \theta\right)^{\frac{1}{z}}} & \\
& \times \int_{z}^{\infty} \frac{J_{1}\left[\xi\left(a^{2}+b^{2}-2 a b \cos \theta\right)^{\frac{1}{2}}\right]}{\left(\xi^{2}-z^{2}\right)^{3}} d \xi
\end{aligned}
$$

with the aid of (2) [setting $\nu=1, \mu=-\frac{1}{2}$, and recalling that $\left.J_{i}(x)=(2 / \pi x)^{t} \sin x\right](5)$ becomes

$\frac{\partial}{\partial a} G(a, b, z)=-\frac{a-b \cos \theta}{a^{2}+b^{2}-2 a b \cos \theta} \cdot \frac{1}{z} \cdot \sin \left[z\left(a^{2}+b^{2}-2 a b \cos \theta\right)^{1}\right]$.

Integrating (6), we get

$G(a, b, z)-G(0, b, z)$

$$
=-\frac{1}{z} \int_{0}^{a} \frac{x-b \cos \theta}{x^{2}+b^{2}-2 b x \cos \theta} \sin \left[z\left(x^{2}+b^{2}-2 x b \cos \theta\right)^{2}\right] d x .
$$

Making a change of variable, $v=\left(x^{2}+b^{2}-2 x b \cos \theta\right)^{2},(7)$ takes the form

$$
G(a, b, z)-G(0, b, z)=\frac{1}{z} S i(b z)-\frac{1}{z} S i\left[z\left(a^{2}+b^{2}-2 a b \cos \theta\right)^{1}\right]
$$

where

$$
S i(x)=\int_{0}^{x} \frac{\sin t}{t} d t
$$

To evaluate $G(0, b, z)$ we note that by $(4)$

$$
G(0, b, z)=\int_{z}^{\infty} \frac{J_{0}(\xi b)}{\xi\left(\xi^{2}-z^{2}\right)^{b}} d \xi .
$$

then

$$
\frac{\partial}{\partial b} G(0, b, z)=-\int_{z}^{\infty} \frac{J_{1}(\xi b)}{\left(\xi^{z}-z^{2}\right)^{2}} d \xi
$$

or with the aid of (2) (setting $\nu=1, \mu=-\frac{1}{2}$ )

$$
\frac{\partial}{\partial b} G(0, b, z)=-\frac{1}{b z} \sin b z .
$$

Integrating (11) with respect to $b$, we get

$$
G(0, b, z)-G(0,0, z)=-\frac{1}{z} \int_{0}^{b} \frac{\sin b z}{b} d b=-\frac{1}{z} S i(b z) .
$$

But since

we obtain

$$
G(0,0, z)=\int_{2}^{\infty} \frac{d \xi}{\xi\left(\xi^{2}-z^{2}\right)^{2}}=\frac{\pi}{2 z},
$$

$$
G(0, b, z)=\frac{\pi}{2 z}-\frac{1}{z} S i(b z)
$$

which when substituted into (8) gives

$$
\left.G(a, b, z)=\frac{\pi}{2 z}-\frac{1}{z} \operatorname{Si}\left[z\left(a^{2}+b^{2}-2 a b \cos \theta\right)\right)^{3}\right] .
$$

It follows from (3), (4), and (15) that

$\int_{z}^{\infty} \frac{J_{0}(a \xi) J_{0}(b \xi)}{\xi\left(\xi^{2}-z^{2}\right)^{2}} d \xi=\frac{\pi}{2 z}-\frac{1}{\pi z} \int_{0}^{\pi} \operatorname{Si}\left[z\left(a^{2}+b^{2}-2 a b \cos \theta\right)^{1}\right] d \theta$ and when $b=a$

$$
\int_{z}^{\infty} \frac{J_{0}^{2}(a \xi)}{\xi\left(\xi^{2}-z^{2}\right)} d \xi=\frac{\pi}{2 z}-\frac{1}{\pi z} \int_{0}^{\pi} S i\left(2 z a \sin \frac{\theta}{2}\right) d \theta
$$

